\title{
Agents in Cyberspace \\ - Towards a Framework for Multi-Agent Systems in Information Discovery
}

\author{
B.C.M. Wondergem \\ Computing Science Institute, University of Nijmegen \\ Nijmegen, The Netherlands \\ P. van Bommel \\ Computing Science Institute, University of Nijmegen \\ Nijmegen, The Netherlands \\ T.W.C. Huibers \\ DOXis \\ Leidschendam, The Netherlands \\ Th.P. van der Weide \\ Computing Science Institute, University of Nijmegen \\ Nijmegen, The Netherlands
}

Published as: B.C.M. Wondergem, P. van Bommel, T.W.C. Huibers, and Th.P. van der Weide. Agents in Cyberspace - Towards a Framework for Multi-Agent Systems in Information Discovery. In Proceedings of the 20th BCS Colloquium on Information Retrieval, IRSG98, Grenoble, France.

Keywords: Multi-Agent Systems, Efficient Document Processing, Distributed Information Retrieval and Filtering, Information Brokering.

\begin{abstract}
This article proposes a formal framework for Multi-Agent Systems in the context of Information Discovery. Information Discovery is a synthesis of Information Retrieval and Information Filtering. The Information Discovery Paradigm is given. In addition, the different types of agents needed in Information Discovery applications are described in terms of the operations they support and the knowledge and information they use. A correct filtering topology, consisting of sound filter paths, is identified. Three fields are identified in which Information Retrieval and Information Filtering benefit from their synthesis: query expansion, query generation or autonomous IR, and profile adaptation.
\end{abstract}


Agents in Cyberspace - Towards a Framework for Multi-Agent Systems in Information Discovery

\section{Introduction}

The amount of information made available through different media is growing rapidly. In parallel, our need for accurate information increases as well. Therefore, for a single user, the quest for relevant information no longer is a sinecure, even with the help of state of the art search engines.

Two main approaches to obtaining relevant information have appeared: Information Retrieval (IR) (see [6]) and Information Filtering (IF). See [1] for an adept comparison of these strongly related plans of attack. In IR, the user formulates his short term information need in the form of a query, which is subsequently processed by a retrieval engine. In IF, long term interests of the user are captured by user profiles, against which descriptions of incoming documents are matched.

The old paradigms of IR and IF, i.e., single user and single resource, have clear shortcomings in a networked setting plagued with an information glut. A new paradigm is needed which consists of a synthesis of the old paradigms for IR and IF and which supports a networked environment, i.e., multiple users and resources. The combination of IR and IF is what we call Information Discovery (ID). As shown later, IR and IF can mutually benefit from their synthesis. Moreover, if IR and IF can be integrated in a single application, the user only has to work with a single system at little or no expense of increased complexity.

The amount of available information has become too large for a single user to cope with properly. To relieve the user's burden, an information broker is introduced as an intermediary between users and resources. The broker aids users in the quest for relevant information. Agent technology is used to develop the information brokers. We adhere to the view of agents as autonomous, intelligent, proactive, reactive, and socially able software programs. ID applications highly require communication, since agents are to solve complex tasks cooperatively, and proactiveness, mainly to relieve the users from taking the initiative. The information broker performs its tasks in cooperation with other agents and only prompts the user when necessary. We will thus consider the ID paradigm from the point of view of multi-agent systems. Much research has been done into agents (see e.g. [5]) and especially in the field of formal logics (see e.g. [10], [4], and [8]).

However, the use of agents in ID has, to this moment, been rather pragmatic and ad hoc. Most of the agents used in ID are not developped from a formal point of view. A more elaborate investigation into the types of agents needed in ID is necessary. The majority of 'agents' used in ID systems does not conform to the notion of agency. Mostly, the principles of communication, intelligence and proactiveness lack completely or only exist in a rudimentary fashion. The Informant ${ }^{1}$, for instance, uses a strictly scheduled form of proactiveness. The users are informed on a pre-set regular interval. The agents that can be created by the Verity $^{2}$ system lack communication and intelligence. In addition, a yet more restricted form of proactiveness is used: the interval is set to one day. Autonomy Agentware's agents ${ }^{3}$ have been made intelligent by the use of neural networks.

Multi-agent systems for ID will be considered from a theoretical basis. In doing this, however, the practical context will not be denied. There are several reasons for the need of a theoretical and formal framework. In the first place, a framework is needed in which multi-agent systems for ID can be designed and defined. Second, the framework is needed to

\footnotetext{
${ }^{1}$ Available from http://informant. dartmouth . edu/

${ }^{2}$ Available from http :// $\mathrm{HH}$.verity .com/

${ }^{3}$ Available from http://ннн.agentнare.com/
} 
analyse, characterise, and compare multi-agent systems through their statical properties. In the third place, we need to go beyond time consuming empirical performance measurements such as recall and precision and move on to the fundamental certainty of logical proofs. The performance and behaviour of ID systems should thus be described on an axiomatic level (see $[2])$.

The goal of this article is to make a first step towards a theoretical framework in which the way ID agents cooperatively make relevance decisions, also called aboutness decisions, can be described, analysed, and compared.

The overview of this paper is as follows. Section 2 provides the ID paradigm. Section 3 describes the types of agents needed in ID. In Section 4, the theoretical framework is provided. Section 5 offers concluding remarks.

\section{Information Discovery}

This section describes the ID paradigm, relates ID to multi-agent systems and gives necessary formal preliminaries.

\subsection{The Information Discovery Paradigm}

An ID system considers three main spaces of interest, as described in [9]: a user space, a resource space and a broker space. See Figure 1 for a schematic overview of the ID paradigm.

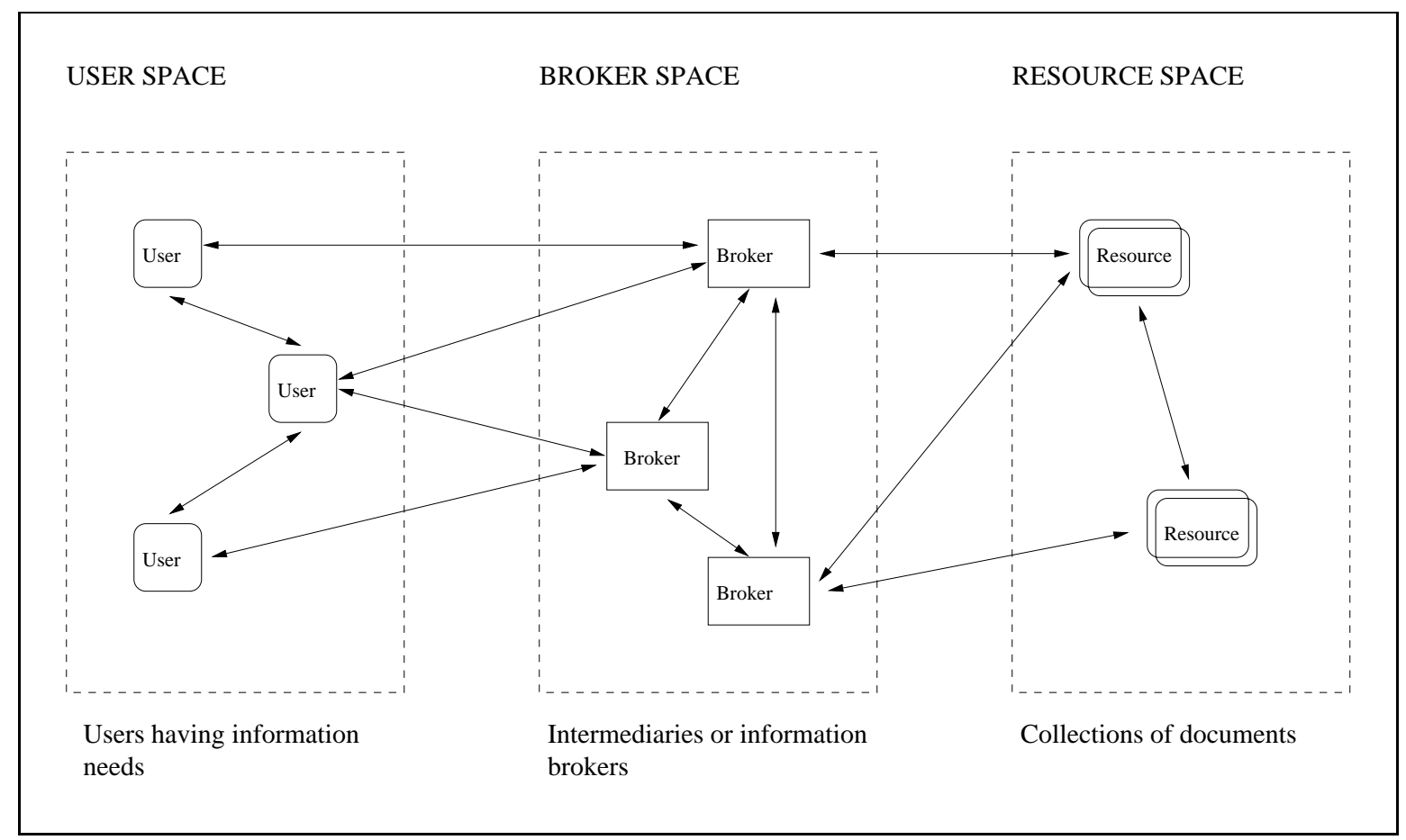

Figure 1: The Information Discovery Paradigm

The user space consists of several users having a number of different long term information needs as well as a short term information need. The information needs are to be satisfied with relevant information, i.e., relevant documents. These documents are drawn from a number of 
Agents in Cyberspace - Towards a Framework for Multi-Agent Systems in Information Discovery

resources, i.e., collections of documents. The relevance estimates of documents with respect to information needs are made by intermediaries, called information brokers. Each user is attached to a number of information brokers, which, in turn, are attached to a number of resources. Information brokers obtain the user's information needs and document characterisations (not necessarily in this order, though), and match these to distinguish relevant from irrelevant documents.

Since information brokers form intermediaries between the users and the resources, direct communication between users and resources is not possible. However, intra-space communication is possible within the three spaces. For example, a number of users that belong to the same department may communicate about their information needs. In addition, information brokers may communicate to complete a complex or time consuming task cooperatively.

\subsection{Information Discovery and Multi-Agent Systems}

A multi-agent system consists of a number of agents and communication channels between them. The entities that constitute the three spaces of the ID paradigm, i.e., users, brokers and resources, are modeled by corresponding types of agents, i.e., user agents, broker agents and resource agents.

Multi-agent systems for Information Discovery (MASID) are a restriction of the general notion of multi-agent systems, i.e., that of a set of agents and their communication channels. The two differences are that agents can only be of the abovementioned types, and that no direct communication between users and resources is possible.

Definition 1 (Multi-agent system for ID) Let $U$ be a set of user agents, $B$ a set of broker agents, and $R$ a set of resource agents. Then a multi-agent system for ID, also called a $M A S I D$, is a tuple $M=\langle U, B, R, C\rangle$, where

- $U, B$, and $R$ are nonempty and mutually disjoint, and

- $C \subseteq(U \cup B \cup R)^{2} \Leftrightarrow((U \times R) \cup(R \times U))$ is a nonempty set of communication channels such that direct communication between user agents and resource agents is not possible.

Example Figure 1 depicts a multi-agent system for Information Discovery, as it adheres to definition 1: by numbering the agents from top to bottum we obtain $\langle U, B, R, C\rangle$, where $U=\left\{u_{1}, u_{2}, u_{3}\right\}, B=\left\{b_{1}, b_{2}, b_{3}\right\}, R=\left\{r_{1}, r_{2}\right\}$, and $C=\left\{\left(u_{1}, u_{2}\right),\left(u_{2}, u_{3}\right),\left(u_{1}, b_{1}\right),\left(u_{2}, b_{1}\right)\right.$, $\left.\left(u_{2}, b_{2}\right),\left(u_{3}, b_{2}\right),\left(b_{1}, b_{2}\right),\left(b_{1}, b_{3}\right),\left(b_{2}, b_{3}\right),\left(b_{1}, r_{1}\right),\left(b_{3}, r_{1}\right),\left(b_{3}, r_{2}\right),\left(r_{1}, r_{2}\right)\right\}$.

\subsection{Formal Preliminaries}

A formal framework requires a formal definition of the descriptor language as well. The descriptor language is used for query and profile formulation and for document characterisation. As all languages in this paper, it is defined in a general way. This is done to abstract from translation issues between agents, needed if they do not speak the same language.

Definition 2 (Descriptor Language) Given a nonempty and finite set of basic elements $K$, the descriptor language, denoted $L_{K}$, is defined as the smallest superset of $K$ such that: if $\alpha \in K$ and $\beta \in K$ then $\neg \alpha \in L_{K},(\alpha \wedge \beta) \in L_{K}$, and $(\alpha \vee \beta) \in L_{K}$. The elements of $L_{K}$ are called descriptors. 
Agents in Cyberspace - Towards a Framework for Multi-Agent Systems in Information Discovery

Example descriptors are (France $\wedge \neg$ wine) and (animals $\vee$ beasts), if the single keywords are drawn from the set of keywords $K$. If those are interpreted as queries, the first states that the user is interested in documents about France but not about wine and the second states that the information need is satisfied by documents about animals or about beasts. Brackets are omitted if not necessary.

The semantics of the descriptor language which is used in the examples, follows the standard interpretations of the logical operators for conjunction and disjunction. In addition, it adheres to the Closed World Assumption for negations. This choice is, however, not crucial.

\section{Types of Agents in Information Discovery}

This section analyses the types of agents used in ID. In addition, a formal representation of these agents is given.

\section{$3.1 \quad$ User agents}

User agents derive user goals, interests and information needs. This process is called user modeling (see [7] for information on the user modeling component within the Profile project). A user agent forms an abstraction of the user, called a user profile, the representation of which is actually worked with. Each user is appointed a number of user agents. This allows for different views on user behaviour.

A user agent enables the user to specify a query, a description of a short term interest, which belongs to a single information need. In addition, it adds abstractions of the (long term) interests of the user to the user profile. Each long term interest corresponds to a distinct information need. The set of all possible information needs is denoted by $\mathcal{N}$. See Figure 2 for a schematic representation of a user agent.

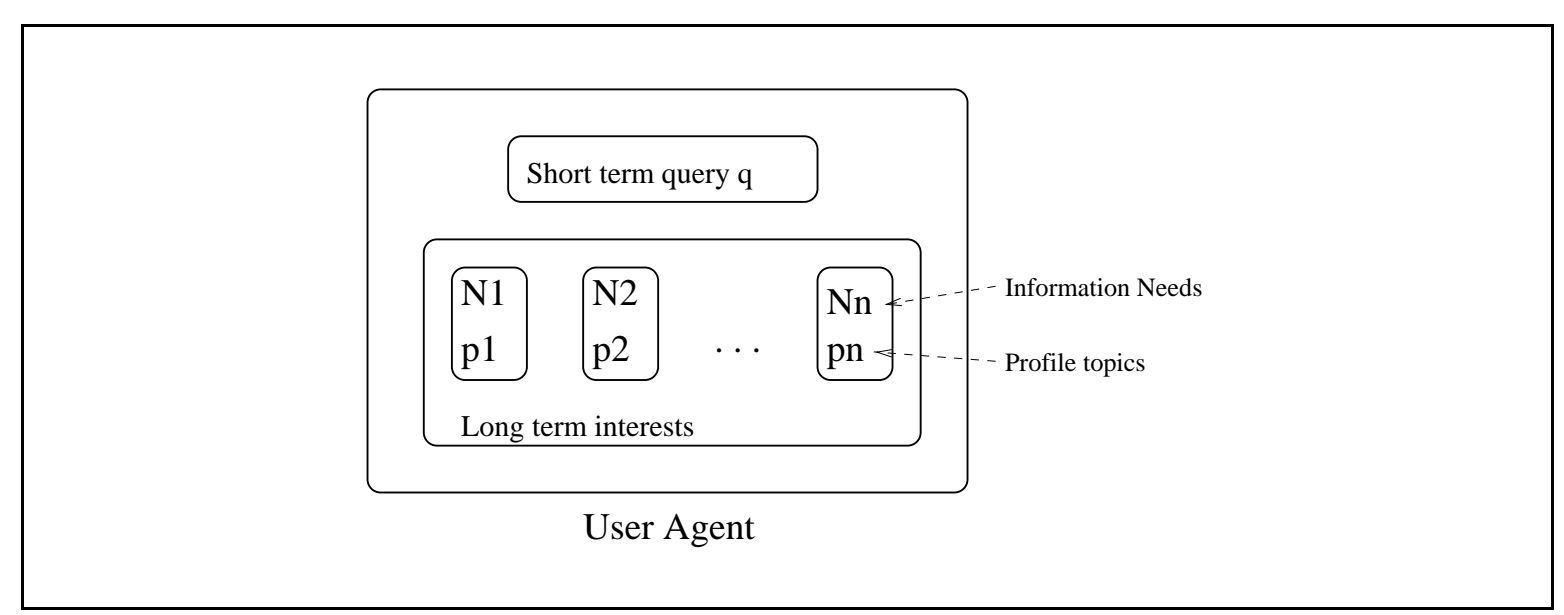

Figure 2: The information contained in a user agent.

Definition 3 (User Agent) A user agent is a tuple $\langle q, P\rangle$, where $q \in L_{K}$ is the user query and $P \subseteq L_{K} \times \mathcal{N}$ is the user profile. The elements of the user profile, called profile topics, combine a descriptor to an information need. 
Agents in Cyberspace - Towards a Framework for Multi-Agent Systems in Information Discovery

Example David works at a software house and spends his leasure time at sea. His long term interests concern computers and internet (information need $N_{\text {comp }}$ ), and wind or waves (information need $N_{\text {sea }}$ ). David has formulated his short term interest in a query about surfing. This is modeled by the following user agent

$u_{\text {david }}=\left\langle\right.$ surfing, $\left\{\left(\right.\right.$ computer $\wedge$ internet, $\left.N_{\text {comp }}\right),\left(\right.$ wind $\vee$ waves, $\left.\left.\left.N_{\text {sea }}\right)\right\}\right\rangle$.

In the semantics of a user agent, distinction is made between the different profile topics. This is done because these may correspond to different information needs and may thus be unrelated. Different profile topics therefore cannot be freely combined. The semantics of a user agent is therefore defined with respect to the view the agent has, i.e., the information need the agent considers or focusses on. Informally speaking, the user agent that considers information need $N$ can only use the corresponding descriptor, i.e., descriptor $p$ from the profile topic $(p, N)$.

Definition 4 (User Semantics) Let $u=\langle q, P\rangle$ be a user agent within multi-agent system $M=\langle U, B, R, C\rangle$, i.e., $u \in U$. Then, the semantics of the user agent in the multi-agent system is defined as:

(i) for every information need $N \in \mathcal{N}: u, N \models^{M} q$

(ii) for every profile topic $(p, N) \in P: u, N \models{ }^{M} p$

The formula $u, N \models^{M} p$ reads: within $u$ 's focus on information need $N$, the descriptor $p$ is valid. Item (i) expresses that the short term query $q$ is valid in all information needs, since it is not clear if it is related to any one of them. This is only a first approximation; in later stages of our research, this assumption will be refined. It should be noted, however, that this assumption leads to the loss of discrepancy between query and long term interests. In particular, with this assumption the query can always be augmented with terms from the long term interests. For certain cases, this is an undesired property which can be overcome by making the communication between agents explicit. This can, for instance, been done by using messages. The user agent may then mold the query and send the result to a broker. The broker, unaware of user descriptors which do not appear in the message, cannot take more terms into account. Item (ii) expresses that for a profile topic $(p, N)$, within $u$ 's focus on $N$, only the corresponding descriptor $p$ is valid.

Example To illustrate the different points of view the user can specify, consider again David from example 3.1. The query surfing is interpreted in 2 different ways, i.e., with respect to the 2 different information needs.

Considering the first information need, that about computers and internet, the following expressions are valid: $u_{\text {david }}, N_{\text {comp }} \models$ surfing and $u_{\text {david }}, N_{\text {comp }} \models$ internet. By using the standard interpretation of logical conjunction, we obtain $u_{\text {david }}, N_{\text {comp }} \models$ (internet $\wedge$ surfing).

On the other hand, considering the second information need, we obtain in a similar way $u_{\text {david }}, N_{\text {sea }} \models($ wind $\wedge$ surfing $) \vee($ wave $\wedge$ surfing $)$ but not $u_{\text {david }}, N_{\text {sea }} \models($ internet $\wedge$ surfing $)$.

\subsection{Resource agents}

The term information source will be used for a collection of documents. Resource agents have access to information sources. By a process called characterising or indexing, resource agents derive document characterisations, abstractions of documents. A resource agent supports the 
Agents in Cyberspace - Towards a Framework for Multi-Agent Systems in Information Discovery

characterisations of the documents in its source. Each information source is accessed by a number of resource agents. This allows for different views on a complex information source. A resource agent accesses information sources, which are modeled as a set of documents. In addition, it is able to deliver document characterisations, which consist of a set of keywords. In this article, we interpreted the characterisation as a conjunction of the individual keywords.

Definition 5 (Resource Agent) Let $D$ be a set of documents and $\chi: D \rightarrow \wp(K)$ a characterisation function. Then, the tuple $\langle\chi, D\rangle$ is a resource agent, where $D$ is called the agent's set of documents and $\chi$ is the agent's document characterisation function.

Example Consider a set of 4 documents about surfing $D=\left\{d_{1}, \ldots, d_{4}\right\}$, where $d_{1}=$ Surfing the Internet, $d_{2}=$ Computer Applications, $d_{3}=$ Wind Surfing in Australia, and $d_{4}=$ Wave Surfing. In addition, consider a characterisation function $\chi$ such that

\begin{tabular}{|l|l|}
\hline Doc $d$ & Characterisation $\chi(d)$ \\
\hline$d_{1}$ & surfing $\wedge$ internet \\
$d_{2}$ & computer $\wedge$ applications \\
$d_{3}$ & wind $\wedge$ surfing $\wedge$ australia \\
$d_{4}$ & wave $\wedge$ surfing \\
\hline
\end{tabular}

Then, the tuple $r_{\text {surf }}=\langle\chi, D\rangle$ is a resource agent.

Similar to user agents, where the view of the agent can be focussed on a specific information need, resource agents can focus on specific documents, and thus have different views as well. This is reflected in the semantics of a resource agent.

Definition 6 (Resource Semantics) Let $r=\langle\chi, D\rangle$ be a resource agent within multiagent system $M=\langle U, B, R, C\rangle$, i.e., $r \in R$. Then,

- for every document $d \in D: r, d \models^{M} \chi(d)$

The sentence $r, d \models^{M} \chi(d)$ describes that when resource agent $r$ considers document $d$, the characterisation of that document, i.e., $\chi(d)$, is valid. Note that in this way, one agent per collection of documents is obtained.

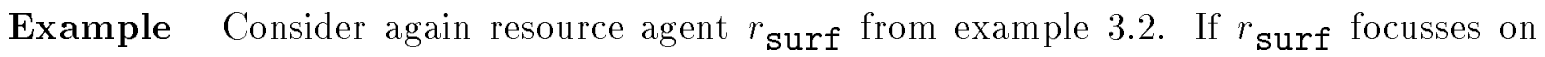
document $d_{1}$, the corresponding characterisation is valid, i.e., $r_{\text {surf }}, d_{1} \models$ surfing $\wedge$ internet. Focussing on document $d_{4}$ results in $r_{\text {surf }}, d_{4} \models$ wave $\wedge$ surfing.

\subsection{Broker Agents}

Broker agents form intermediaries between user agents and resource agents. They act as information brokers, providing users with relevant information. Broker agents match document characterisations with user profiles or queries to establish degrees of relevance of documents with respect to user interests.

Matching is modeled by two operations of the broker agent: aboutness and anti-aboutness. Aboutness states when a descriptor is about another descriptor. Anti-aboutness describes when a descriptor is non-about another descriptor. Matching can be applied in the context of IF or of IR. In IF, a document profile is matched against several user or group profiles. In IR, a number of document characterisations are matched against a specific query. 
Agents in Cyberspace - Towards a Framework for Multi-Agent Systems in Information Discovery

Definition 7 (Broker Agent) A broker agent is a tuple $\langle\triangleright, \triangleright\rangle$, where $\triangleright \subseteq L_{K} \times L_{K}$ is the agent's aboutness relation and $\otimes \subseteq L_{K} \times L_{K}$ is the agent's anti-aboutness relation. If $b=\langle\triangleright, \triangleright\rangle$ is a broker agent, then $\triangleright_{b}$ denotes $\triangleright$ and $\triangleright_{b}$ denotes $\triangleright$.

Example A naive overlap broker can be specified in our framework as $b_{\text {naive }}=\langle$ overlap, disjoint , where

- overlap $=\{(\phi, \psi) \mid \operatorname{PosAt}(\phi) \cap \operatorname{PosAt}(\psi) \neq$ ? $\}$

- disjoint $=\{(\phi, \psi) \mid \operatorname{Pos} A t(\phi) \cap \operatorname{Pos} A t(\psi)=?\}$

assuming the function PosAt $: L_{K} \rightarrow K$, where PosAt $(\phi)$ gives the set of atoms (keywords) that appear possitively in $\phi$.

The aboutness relations of the broker agents are relations on the descriptor language. A particular instance of the aboutness relation holds in an agent iff that instance is part of the agent's aboutness relation.

Definition 8 (Broker Semantics) Let $b=\langle\triangleright, \triangleright\rangle$ be a broker agent and $M=\langle U, B, R, C\rangle$ a multi-agent system, such that $b \in B$. Then,

- $b \models^{M} \phi \triangleright \psi \Leftrightarrow^{\operatorname{def}}(\phi, \psi) \in \triangleright_{b}$

- $b \models^{M} \phi \otimes \psi \Leftrightarrow \Leftrightarrow^{\mathrm{def}}(\phi, \psi) \in \otimes_{b}$

Example The naive overlap broker of the previous example makes the following aboutness

decision: $b_{\text {naive }} \models$ wind $\wedge$ surfing $\wedge$ Hawaii $\triangleright$ wave $\wedge$ surfing $\wedge$ sea. However, for reasons of naivety, it also makes the following statement $b_{\text {naive }} \models$ internet $\wedge$ surfing $\triangleright$ wind $\wedge$ surfing. The overlap broker can be made less naive by introducing a knowledge base containing additional (domain) knowledge. To incorporate the knowledge base, the relations overlap and disjoint have to be redefined using the information in the knowledge base, for instance, synonyms.

It should be noted that the broker's aboutness relation is symmetric with respect to user queries and document contents. That is, the origin of initiative, i.e. IR or IF, is not taken into account by a single aboutness relation. If a discrepancy is to be made, however, two different brokers can be used. The difference can then be obtained by using the first broker's aboutness relation in the case of IR and the second broker's aboutness relation in the case of IF.

\section{Multi-Agent Systems in ID}

This section formalizes the framework for multi-agent systems in Information Discovery (Subsection 1) and elaborates on the two major tasks of the system: Information Retrieval (Subsection 2) and Information Filtering (Subsection 3).

\subsection{Formalizing the Framework}

The semantics of a MASID $M$ is captured by a binary relation $\models^{M}$ stating the validity of formulae in agents. The left hand side of this relation denotes an agent. In the case of user and resource agents, this includes a point of view. A uniform agent language is defined to capture this. The right hand side of the relation contains a formula. Formulas are either 
Agents in Cyberspace - Towards a Framework for Multi-Agent Systems in Information Discovery

descriptors, in the case of user and resource agents, or, in the case of broker agents, (anti-) aboutness statements. This is captured in the aboutness language, which is defined shortly.

Let $\mathcal{D}$ denote the set of all documents considered in a multi-agent system, i.e., the union of all the resource agents' document sets.

Definition 9 (Agent Language) Let $M=\langle U, B, R, C\rangle$ be a multi-agent system. The agent language, denoted $\mathcal{L}_{\text {Agent }}$, is defined by:

$$
\mathcal{L}_{\text {Agent }}=(U \times \mathcal{N}) \cup B \cup(R \times \mathcal{D})
$$

A uniform language is to be defined, capturing all possible formulae agents support. This language, called the aboutness language, consists of the descriptor language, since user and resource agents support descriptors, and aboutness and anti-aboutness statements.

Definition 10 (Aboutness Language) Let $L_{K}$ be a descriptor language. Then the aboutness language, denoted $\mathcal{L}_{\mathrm{About}}$, is the smallest superset of $L_{K}$ such that: if $\phi, \psi \in L_{K}$ then $\phi \triangleright \psi \in \mathcal{L}_{\text {About }}$ and $\phi \triangleright \psi \in \mathcal{L}_{\text {About }}$.

The semantics of a multi-agent system for Information Discovery is now given as a relation between the agent language and the aboutness language. It expresses when an element of the aboutness language is valid in an agent, i.e., an element of the agent language.

Definition 11 (Semantics of MASID) Let $M=\langle U, B, R, C\rangle$ be a MASID. The semantics of $M$ is given by the relation $\models^{M} \subseteq \mathcal{L}_{\text {Agent }} \times \mathcal{L}_{\text {About }}$ for which

- every agent supports some semantics for descriptors,

- every user agent supports the User Semantics (Definition 4),

- every resource agent supports the Resource Semantics (Definition 6), and

- every broker agent supports the Broker Semantics (Definition 8).

Broker agents can be characterized by the way in which they make aboutness decisions in a network of other broker agents. Several types of broker agents are identified.

Definition 12 (Types of Broker Agents) Let $M=\langle U, B, R, C\rangle$ be a MASID. Furthermore, define $C_{b}=\left\{b^{\prime} \in B \mid\left(b^{\prime}, b\right) \in C\right\}$, i.e., all the broker agents $b$ can communicate with. Broker agent $b \in B$ is called

\begin{tabular}{|ll|}
\hline & $b \models^{M} \phi \triangleright \psi$ holds if \\
\hline unanimous & $\forall b^{\prime} \in C_{b}: b^{\prime} \models^{M} \phi \triangleright \psi$ \\
stubborn & $\forall b^{\prime} \in C_{b}: b^{\prime} \models^{M} \phi \triangleright \psi$ \\
optimistic & $\forall b^{\prime} \in C_{b}: b^{\prime} \nvdash^{M} \phi \triangleright \psi$ \\
a lawyer & $\forall b^{\prime} \in C_{b}: b^{\prime} \nvdash^{M} \phi \triangleright \psi$ \\
typical & $\exists b^{\prime} \in C_{b}: b^{\prime} \models^{M} \phi \triangleright \psi$ \\
gambling & $\neg \exists b^{\prime} \in C_{b}: b^{\prime} \models^{M} \phi \triangleright \psi \vee b^{\prime} \models^{M} \phi \triangleright \psi$ \\
\hline
\end{tabular}

IRSG98 
Agents in Cyberspace - Towards a Framework for Multi-Agent Systems in Information Discovery

Example In preparing a case, a lawyer does not want to miss possibly relevant material. This is reflected in the definition of a broker agent that is a lawyer. A form of meta-search, i.e., merging the results of a number of brokers, is obtained if a typical broker is used. All documents which are considered relevant by at least one broker are rendered by a typical broker. An example of a typical broker is MetaCrawler ${ }^{4}$. A unanimous broker is best applied if certainty of relevancy is required. A gambling broker can be used in a filtering context to deliver a mix of 'randomly chosen' documents. It can also be used for system enhancements: by examining the documents a gambling broker passes on, one gaines insight in the shortcomings of the brokers that did not know how to evaluate those documents. In an ideal situation, the users are able to specify what type(s) of brokers should assist them in the quest for relevant information.

Each careful broker agent is also a typical broker agent. If a broker agent does not communicate with other broker agents, it is unanimous, stubborn, a lawyer, optimistic, and gambling.

The differences in relative power, i.e., coverage of aboutness decisions, of broker agents can be exploited for efficient Information Discovery, as shown later. The comparison between broker agents with respect to their relative power is modeled by an embedment relation.

Definition 13 (Embedded Broker Agents) Let $M=\langle U, B, R, C\rangle$ be a multi-agent system and let $b, b^{\prime} \in B$ and $q \in L_{K}$.

- Broker agent $b$ is embedded in $b^{\prime}$, denoted $b \prec b^{\prime}$, iff every aboutness decision of $b$ is also made by $b^{\prime}: \triangleright_{b} \subseteq \triangleright_{b^{\prime}}$, i.e., $\forall \phi, \psi \in L_{K}: b \models^{M} \phi \triangleright \psi \Rightarrow b^{\prime} \models^{M} \phi \triangleright \psi$.

- The broker-query pair $b(q)$ is left embedded in $b^{\prime}\left(q^{\prime}\right)$, denoted $b(q) \prec_{L} b^{\prime}\left(q^{\prime}\right)$, iff every aboutness decision of $b$ regarding $q$ as rightmost part is also made by $b^{\prime}$ regarding $q^{\prime}$, i.e., $\forall \phi \in L_{K}: b \models^{M} \phi \triangleright q \Rightarrow b^{\prime} \models^{M} \phi \triangleright q^{\prime}$.

Lemma 4.1 The relations $\prec$ and $\prec_{L}$ are reflexive, transitive, and not necessarily connected.

Lemma 4.2 Let $b, b^{\prime} \in B$ be broker agents in multi-agent system $M=\langle U, B, R, C\rangle$. Then, broker $b$ is embedded in broker $b^{\prime}$, i.e., $b \prec b^{\prime}$, iff for every query $q \in L_{K}$ broker $b$ is left-embedded in $b^{\prime}$, i.e., $\forall q \in L_{K}: b(q) \prec_{L} b^{\prime}(q)$.

Lemma 4.3 Let $b$ be a unanimous broker agent connected to $b_{1}, \ldots, b_{n}$. Then, broker $b$ is embedded in all the attached brokers, i.e., for all $1 \leq i \leq n: b \prec b_{i}$.

Let $b$ be a typical broker agent connected to $b_{1}, \ldots, b_{n}$. Then, the attached brokers are all embedded in broker $b$, i.e., for all $1 \leq i \leq n: b_{i} \prec b$.

In processing a query, first a large (with respect to the embedment relation) broker having a cheaply evaluable aboutness relation is used to quickly discard of many irrelevant documents. After this, a smaller broker agent (probably with a more expensive aboutness relation) is used to produce the eventual outcome. Potentially, this cuts down expenses drastically. Huibers and Denos use a similar approach in [3] to obtain an ordening on documents.

The next subsection focusses on Information Retrieval issues within a MASID. The subsequent subsection considers Information Filtering issues.

\footnotetext{
${ }^{4}$ Available from http://metacrarler.cs.rashington . edu/
} 
Agents in Cyberspace - Towards a Framework for Multi-Agent Systems in Information Discovery

\subsection{Issues of Information Retrieval in ID}

In the scenario for Information Retrieval in a MASID, a user agent sends a query to a number of broker agents, which, in turn, send requests for documents to resource agents, and, upon receipt of those documents, match document profiles with the query and send relevant documents back to the user agent for rendering.

The result of a broker agent processing a user query in the set of documents of a resource agent, is the set of documents of which the characterisation is about the query according to the broker's aboutness relation. That is, if $u=\langle q, P\rangle$ is a user agent, $b=\langle\triangleright, \triangleright\rangle$ is the broker agent, and $r=\langle\chi, D\rangle$ is a resource agent:

$$
\text { result }(u, b, r)=\left\{d \in D \mid b \models^{M} \chi(d) \triangleright q\right\}
$$

Example Consider the agents from previous examples. We have result $\left(u_{\text {david }}, b_{\text {overlap }}, r_{\text {surf }}\right)=$ $\left\{d \in\left\{d_{1}, \ldots, d_{4}\right\} \mid b_{\text {overlap }} \models^{M} \chi(d) \triangleright\right.$ surfing $\}=\left\{d_{1}, d_{3}, d_{4}\right\}$.

For reasons of efficiency, a broker agent that supports a competent aboutness relation at high costs, can be preceded by a less restrictive and rather cheap broker. We call this process serial composition, and it can, of course, be repeated several times. One and the same document characterization is matched by a number of increasingly complex broker agents. The series of brokers involved is called a broker filter path.

Definition 14 (Broker Filter Path) Let $b_{1}, \ldots, b_{n}$ be broker agents. Then, for every query $q$, the sequence $b_{1}(q), \ldots, b_{n}(q)$ is called a broker filter path. The broker filter path is sound iff $b_{n} \prec \ldots \prec b_{1}$, i.e., the broker agents are increasingly restrictive.

Lemma 4.4 Let $b$ be a sound broker filter path of which $b_{n}$ is the last broker agent. Then, $\bigcap_{b_{i} \in b} \triangleright_{b_{i}}=\triangleright_{b_{n}}$.

The simple scenario for IR can, of course, be augmented. In the remainder of this subsection, we discuss query expansion, user profile adaptation and autonomous IR or query generation.

One of the benefits of the synthesis of IR and IF is that the user profile forms a naturally personalized context to expand the user query in. The user agent can expand the user query with respect to its user profile, i.e., all the profile topics available, obtaining a set of expanded queries which are sent to a broker agent for processing. This form of expansion is called profile expansion.

A user profile consists of several unrelated information needs. Furthermore, the user query is formulated in the light of a single information need. Instead of expanding the query to the complete user profile, the user agent can also expand the query to the corresponding profile topic, i.e., the profile topic that belongs to the information need in the light of which the user query was formulated. To formalize this, we assume a similarity function, e.g., $\sigma: L_{K} \times L_{K} \rightarrow[0, . ., 1]$, where the similarity between descriptors $\phi$ and $\psi$ is larger iff $\sigma(\phi, \psi)$ is higher. The procedure now is first to find the profile topic $(p, N)$ that maximizes $\sigma(q, p)$, and then to expand to this topic only. This form of expansion is called topic expansion.

Query expansion can also be used for user aided disambiguation of query terms. In example 3.1 we saw that a query term has different interpretations in different profile topics. To assess the correspondence of the profile topics to the query, the query is expanded with respect to all the profile topics. User relevance feedback on the retrieved documents then indicates the desired interpretation, i.e., the corresponding profile topic. 
Agents in Cyberspace - Towards a Framework for Multi-Agent Systems in Information Discovery

Both profile and topic expansion only take the user's own profile into account. A form of widened expansion is obtained if the user profiles of related users are taken into account as well. In order to contact the related users, communication channels in the multi-agent system can be followed. Query expansion can now take place with respect to a set of user profiles. In expanding the query to the individual profiles of this set, profile or topic expansion can be used.

Another augmentation of the simple IR scenario is the adaptation of user profiles. Three moments for this can be identified. First, when the user specifies a new query. The similarity function can be applied to obtain the most similar profile topic which can then be adapted according to the query. Second, on the rendering of the relevant documents, the characterisations of the documents as well as the query itself can be used for profile adaptation. A more nuanced approach is acchievable if relevance feedback is given, i.e., if the user is able to explicitly mark some documents as (non-) relevant. The document characterisations of these documents only are then used for profile adaptation. Thus, by exploiting user queries for the adaptation of user profiles, IF benefits from the synthesis with IR.

Autonomous IR is the third advantage of the synthesis between IR and IF. Whereas in the simple IR scenario the initiative is in the hands of the user, user agents, being proactive, can also start an IR task themselves, thus performing autonomous IR. In order to do this, the user agent generates a query which is subsequently sent to a broker agent. The user agent generates queries on the basis of the user profile, thus serving the user in his information needs.

\subsection{Issues of Information Filtering in ID}

The matching of user profiles with document characterisations, i.e., computing the result sets, is the same in IF as in IR. Again, the result sets are computed with the (anti-)aboutness relations of broker agents. The differences in filtering with retrieval are that in IF the initiative is in the hands of the documents, and, that a topology of user agents can be exploited for efficient filtering. Since the switch of initiative cannot be modeled properly in our static framework, we will focus on the topology.

Example Figure 3 shows an example of a filtering topology: profiles higher up in the hierarchy are less specific than the lower ones. Incoming documents are sent to the root of the hierarchy and find their way down to user agents by the normal matching procedures.

In a similar way to obtaining efficient brokers, i.e., by serial composition, efficient filtering can be achieved by serially combining user profiles. Starting, again, in a bottom up fashion, the user profiles are preceded by less specific group profiles. Matching, then, starts with rather general group profiles, gradually proceeds through more complex group profiles, and, finally, ends in the user profiles.

As with serially composed broker agents, care must be taken in the way in which the profiles are actually combined. The series of profiles must be of increasing complexity to guarantee that no relevant documents are discarded of at an intermediate stage.

Definition 15 (User Filter Path) Let $q_{1}, \ldots, q_{n} \in L_{K}$ be queries and let $b \in B$ be a broker agent. Then, the sequence $b\left(q_{1}\right), \ldots, b\left(q_{n}\right)$ is called a user filter path. The user filter path is sound iff $\forall 1 \leq i<n: b\left(q_{i+1}\right) \prec_{L} b\left(q_{i}\right)$.

Example Assume that every user agent in Figure 3 is attached to a naive overlap broker 


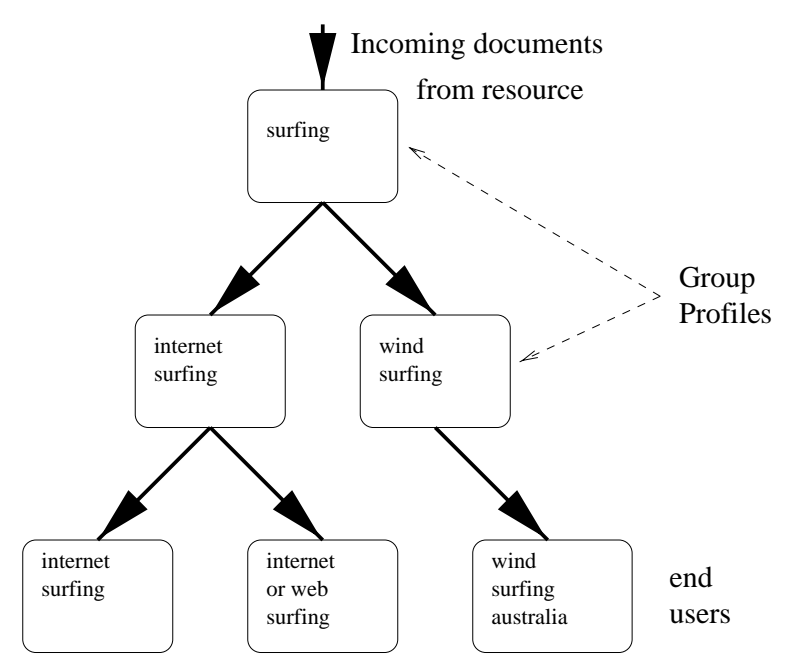

Figure 3: Filtering Topology

agent. Then, all user filter paths in Figure 3 are sound user filter paths. In the opposite direction they are not sound user filter paths.

Now, information is needed to derive the purpose of a communication channel. Several types of channels can be defined between user agents. For instance, semantical channels like a channel from a student to a teacher, or from employer to employee, or, structural channels used for filtering. In order to be able to select the proper channels, the channels are labeled with their function. That is, a channel is a 3-tuple of which the components denote the source agent, the target agent, and the type of the channel, respectively. That is, a channel is an element of $\mathcal{L}_{\text {Agent }} \times \mathcal{L}_{\text {Agent }} \times \mathcal{T}$, where $\mathcal{T}$ is a set of channel types. The type for filter channels is filter.

The scenario's for query expansion, as described in the previous section, can now be refined by using only channels of certain types. For instance, widened expansion can be performed with respect to a whole company, for example by using the company channels, or with respect to a single department, i.e., by only using the R\&D-department channels.

Thus far, we have described two ways to improve the efficiency of a MASID: serial composition of increasingly complex broker agents or user profiles. In serially composed brokers the query remains the same, and in serially combined user profiles there is only one broker agent. To generalise this, a sound filter path, consisting of a number of brokers and queries, is one that does not discard of any documents at intermediate stages that are considered relevant in a later stage. This leads to a more general definition of (soundness) of a filter path.

Definition 16 (General Filter Path) Let $b_{1}, \ldots, b_{n} \in B$ be broker agents, and $q_{1}, \ldots, q_{n} \in$ $L_{K}$ be queries. Then, the sequence $b_{1}\left(q_{1}\right), \ldots, b_{n}\left(q_{n}\right)$ is called a general filter path. The general filter path is sound iff every broker-query pair is left embedded in the previous broker-query pair, i.e., $\forall 1 \leq i<n: b_{i+1}\left(q_{i+1}\right) \prec_{L} b_{i}\left(q_{i}\right)$.

Lemma 4.5 If a broker filter path is sound, it is also a sound general filter path. If a user filter path is sound, it is also a sound general filter path. 
Agents in Cyberspace - Towards a Framework for Multi-Agent Systems in Information Discovery

In order to properly define a correct filtering topology, we single out the channels that are used for filtering. We assume that serially composed broker agents and user filter paths only are constructed with channels of type filter. The filtering channels are defined by

$$
C_{\mathcal{F}}=\left\{\left\langle a, a^{\prime}, t\right\rangle \in C \mid t=\text { filter }\right\}
$$

The definition of a filtering topology reflects that the filtering topology should lead documents directly down to user agents and that filter paths are correctly constructed in terms of form and soundness. The definition reflects that an acyclic topology is needed, consisting of series of user agents such that together with their associated broker agents they form sound filter paths.

Definition 17 (Filtering Topology) A multi-agent system $M=\langle U, B, R, C\rangle$ is said to have a filtering topology iff

- $C_{\mathcal{F}}$ describes a forest, i.e., a set of trees, and

- every user agent participating in $C_{\mathcal{F}}$ is attached to a broker agent, and

- every filter path in $M$ is sound.

We now have tools to formally decide if a multi-agent system for Information Discovery adheres to a filtering topology. If not, we are able to inspect the filter paths to locate the shortcomings.

\section{Conclusions}

This article provides a formal framework in which Multi-Agent Systems for Information Discovery (MASID) can be statically described, analysed, and compared. Multi-agent systems for information discovery can be compared with respect to their statical constructional properties, such as the number of agents used of the different types, the types of channels that are possible, and the types of broker agents used. It can be checked, for example, if a MASID Adheres to a sound filtering topology. First, the Information Discovery paradigm was stated, identifying the types of agents needed. These agents as well as Multi-Agent Systems for Information Discovery were described in more detail and formalised.

Further research will focus on implementing a prototype of the Profile (see e.g. [9]) system and describing it in the framework developed. To this end, the framework has to be augmented with, for instance, domain knowledge and a richer descriptor language. In addition, the dynamic aspects of MASIDs also form a main topic for further research.

\section{References}

[1] N. Belkin and W. Croft. Information filtering and information retrieval: Two sides of the same coin? Communications of the ACM, 35(12):29-38, Dec. 1992.

[2] T. Huibers. An Axiomatic Theory of Information Retrieval. PhD thesis, Department of Computer Science, Utrecht University, November 1996. 
Agents in Cyberspace - Towards a Framework for Multi-Agent Systems in Information Discovery

[3] T. Huibers and N. Denos. A qualitative ranking method for logical information retrieval models. Technical Report RAP95-005, Groupe MRIM of the Laboratoire de Génie Informatique, Grenoble, France, Aug. 1995.

[4] T. Huibers and B. Linder. Formalising Intelligent Information Retrieval Agents. In Proceedings of the 18th British Computer Society Annual Information Retrieval Colloqium, pages 125-143, Manchester, England, 1996. Manchester Metropolitan University.

[5] S. Marsh. A Community of Autonomous Agents for the Search and Distribution of Information in Networks. In J. Furner and D. Harper, editors, Proceedings of the 19th BCS-IRSG Colloquium on IR Research, Aberdeen, Scotland, April 1997.

[6] C. v. Rijsbergen. Information Retrieval. Butterworths, London, United Kingdom, 2nd edition, 1979 .

[7] J. Simons. Using a semantic user model to filter the world wide web proactively. In A. Jameson, C. Paris, and C. Tasso, editors, Proceedings of the sixth international Conference UM97. SpringerWien New York, 1997.

[8] B. van Linder. Modal Logics for Rational Agents. PhD thesis, Department of Computer Science, Utrecht University, The Netherlands, June 1996.

[9] B. Wondergem, P. v. Bommel, T. Huibers, and T. v. d. Weide. Towards an Agent-Based Retrieval Engine. In J. Furner and D. Harper, editors, Proceedings of the 19th BCS-IRSG Colloquium on IR research, pages 126-144, Aberdeen, Scotland, April 1997.

[10] M. Wooldridge and N. Jennings. Intelligent Agents: Theory and Practice. Knowledge Engineering Review, 10(2):115-152, 1995. 